\title{
The YARK Theory of Gravity Can Reproduce Neither the LIGO "GW150914 Signal", nor the Other LIGO Detections of Gravitational Waves
}

\author{
Christian Corda \\ International Institute for Applicable Mathematics and Information Sciences, B. M. Birla Science Centre, \\ Adarshnagar, Hyderabad 500063, India; cordac.galilei@gmail.com
}

Received: 3 October 2018; Accepted: 25 October 2018; Published: 1 November 2018

\begin{abstract}
We show that, based on important reasons, differently from some recent claim in the literature, the YARK theory of gravity can reproduce neither the LIGO "GW150914 signal", nor the other LIGO detections of gravitational waves (GWs).
\end{abstract}

Keywords: gravitational waves; interferometers; equivalence principle; YARK theory of gravity

The YARK (from the initials of the proper surnames of its authors) theory of gravity was originally proposed by T. Yarman in Foundation of Physics [1]. After that, various papers on the YARK theory of gravitation were published by T. Yarman and collaborators (O. Yarman, A. L. Kholmetskii and M. Arik; we refer to them as the YARK group) [2-10]. This is a bit surprising, because the YARK theory of gravity is a non-metric theory, and, as we have rigorously shown in our recent paper [11] (but this issue is well known; see for example Will's review [12]), it macroscopically violates Einstein's equivalence principle, which has today strong, indisputable, empiric evidence [11,12]. On the other hand, despite the YARK group claims that their theory is in agreement with various experimental and/or observational results [3-10], we have shown that it cannot explain the Mössbauer rotor experiment [13], contrary to their claims in [5]. In fact, in [13,14], we have shown that in the Mössbauer rotor experiment, an additional effect of clock synchronization is present, which has not been considered by the YARK group in [5]. Remarkably, our result on the Mössbauer rotor experiment can be considered a new proof of Einstein's general theory of relativity, and, for this reason, it has been recently awarded Honorable Mention in the 2018 Essay Competition of the Gravity Research Foundation [15].

For the sake of completeness, we add some detail on the YARK theory of gravity. It is basically based on the energy conservation law [5]. In such an approach, space-time is Minkowskian-like, and the Christoffel symbols are equal to zero. In addition, the diagonal metric coefficients are multiplied by a conformal factor that depends on the "static gravitational binding energy" of a test particle [5]. Thus, the YARK group claims that their theory combines both metric and dynamical approaches [5]. This seems an apparent contradiction with our above statement that "the YARK theory of gravity is a non-metric theory". However, the key point is that, instead of the geodesic motion in a curved space-time, the YARK theory shows a dynamical equation of motion for a test particle in a flat space-time governed by the force resulting from the spatial variation of the gravitational binding energy [5]. In fact, in the common language of gravitational theorists, a metric theory is defined as being a theory that satisfies Einstein's equivalence principle [12], which, in turn, implies that gravitation must be a "curved space-time" phenomenon [12]. This means that the effects of gravitation are completely equivalent to the effects of living in a curved space-time [12]. Thus, we have at least two issues in the YARK theory of gravity that are in strong contrast with Einstein's equivalence principle. The first is that space-time is flat [5]. The second is the statement that the gravitational energy can be localized [5]. For this second point, we recall that another consequence of Einstein's equivalence principle is indeed that one can always find in any given locality a reference frame (the local Lorentz 
reference frame) in which all local gravitational fields are null. No local gravitational field means no local gravitational energy-momentum and, in turn, no stress-energy tensor for the gravitational field $[11,16]$.

Another recent claim of the YARK group is that their theory can reproduce the LIGO "GW150914 signal", as well as the other recent LIGO GW detections [10]. Here, we show that this claim is not correct. In fact, we see that in [10] (in the paper [10], C.B. Marchal was an additional author), the YARK group claims that, while GWs are not present in their theory, the same theory admits a difference $\triangle \varphi$ of the phase shift of light due to electromagnetic radiation incoming from the coalescence of super-massive bodies in a distant binary system; see Equations (11) and (14) in [10]. In the YARK theory, such a value $\triangle \varphi$ is the same for both arms of the interferometer, and the ratio $\left|\frac{\Delta \varphi}{\varphi}\right|$, where $\varphi$ is the phase of the interferometer light beam, should be of the same order of magnitude of the GW150914 signal [10]. However, the key point is that LIGO and the other interferometric GW detectors operate in a differential mode. In other words, the total phase shift of light, which represents the output of LIGO, is given by the difference between the phase shifts of light of each arm of the interferometer; see for example $[17,18]$. Thus, if the value $\triangle \varphi$ in the YARK theory is the same for both arms of the interferometer, the total output of LIGO must be null. In other words, if the YARK theory is correct, LIGO cannot detect signals.

On the other hand, in [10], the YARK group claims that the relative phase shift that is admitted by their theory has an electromagnetic origin. However, this is in contrast with the event GW170817 [19]. In fact, in such an event, there was a time delay in the detection of the electromagnetic counterpart of the LIGO signal [19]. Thus, if the YARK theory is correct, it should have been impossible to have such a delay.

Resuming, in this work, it has been shown that, based on important reasons, differently from the claims in [10], the YARK theory of gravity can reproduce neither the LIGO "GW150914 signal", nor the other LIGO detections of GWs. If we add that the YARK theory of gravity violates Einstein's equivalence principle [11] and is in disagreement with the Mössbauer rotor experiment [14-16], we conclude that this theory is largely disfavored.

Funding: There is no extra funding.

Acknowledgments: The author thanks the referees of this paper for their useful comments.

Conflicts of Interest: The author declares no conflict of interest.

\section{References}

1. Yarman, T. The end results of general relativity theory via just energy conservation and quantum mechanics. Found. Phys. Lett. 2006, 19, 675-693. [CrossRef]

2. Kholmetskii, A.; Yarman, T.; Yarman, O.; Arik, M. Mössbauer experiments in a rotating system, Doppler effect and the influence of acceleration. Eur. Phys. J. Plus 2018, 133, 261. [CrossRef]

3. Yarman, T.; Kholmetskii, A.L. Sketch of a cosmological model based on the law of energy conservation. Eur. Phys. J. Plus 2013, 128, 8. [CrossRef]

4. Yarman, T.; Kholmetskii, A.L.; Arik, M. Mössbauer experiments in a rotating system: Recent errors and novel interpretation. Eur. Phys. J. Plus 2015, 130, 191. [CrossRef]

5. Kholmetskii, A.L.; Yarman, T.; Arik, M. Comment on "Interpretation of Mössbauer experiment in a rotating system: A new proof by general relativity". Ann. Phys. 2015, 363, 556-558. [CrossRef]

6. Kholmetskii, A.L.; Yarman, T.; Yarman, O.; Arik, M. Response to "The Mössbauer rotor experiment and the general theory of relativity" by C. Corda. Ann. Phys. 2016, 374, 247-254. [CrossRef]

7. Yarman, T.; Arik, M.; Kholmetskii, A.; Yarman, O. Super-massive objects in Yarman-Arik-Kholmetskii (YARK) gravitation theory. Can. J. Phys. 2016, 94, 271-278. [CrossRef]

8. Arik, M.; Yarman, T.; Kholmetskii, A.; Yarman, O. Yarman's approach predicts anomalous gravitational bending of high-energy gamma-quanta. Can. J. Phys. 2016, 94, 616-622. [CrossRef]

9. Yarman, T.; Kholmetskii, A.; Arik, M.; Yarman, O. Pound-Rebka result within the framework of YARK theory. Can. J. Phys. 2016, 94, 558-562. [CrossRef] 
10. Yarman, T.; Kholmetskii, A.L.; Yarman, O.; Marchal, C.B.; Arik, M. LIGO's “GW150914 signal” reproduced under YARK theory of gravity. Can. J. Phys. 2017, 95, 963-968. [CrossRef]

11. Corda, C. Comment on Eur. Phys. J. Plus 133, 261 (2018) by Kholmetskii et al. (The YARK theory of gravity is wrong). arXiv 2018, arXiv:1508.02240.1808.09815.

12. Will, C.M. The confrontation between general relativity and experiment. Living Rev. Relativ. 2014, 17, 4. [CrossRef] [PubMed]

13. Corda, C. The Mössbauer rotor experiment and the general theory of relativity. Ann. Phys. 2016, 368, $258-266$. [CrossRef]

14. Corda, C. Interpretation of Mössbauer experiment in a rotating system: A new proof for general relativity. Ann. Phys. 2015, 355, 360-366. [CrossRef]

15. Corda, C. New proof of general relativity through the correct physical interpretation of the Mössbauer rotor experiment. arXiv 2018, arXiv:1805.06228.

16. Misner, C.W.; Thorne, K.S.; Wheeler, J.A. Gravitation; W. H. Freeman: San Francisco, CA, USA, 1973.

17. Saulson, P.R. Fundamentals of Interferometric Gravitational Wave Detectors; World Scientific: Singapore, 1994.

18. Corda, C. Interferometric detection of gravitational waves: The definitive test for General Relativity. Int. J. Mod. Phys. D 2009, 18, 2275-2282. [CrossRef]

19. Alexander, K.D.; Berger, E.; Fong, W.; Williams, P.K.G.; Guidorzi, C.; Margutti, R.; Metzger, B.D.; Annis, J.; Blanchard, P.K.; Brout, D.; et al. The electromagnetic counterpart of the binary neutron star merger LIGO/VIRGO GW170817. VI. Radio constraints on a relativistic jet and predictions for late-time emission from the kilonova ejecta. Astrophys. J. Lett. 2017, 848, L21. [CrossRef]

(C) 2018 by the author. Licensee MDPI, Basel, Switzerland. This article is an open access article distributed under the terms and conditions of the Creative Commons Attribution (CC BY) license (http:/ / creativecommons.org/licenses/by/4.0/). 